\title{
Virtual Branch Analysis of Symbol Error Rate for OSTBC MIMO Systems with Generalized Selection Combining Using MPSK and MQAM Modulation Schemes
}

\author{
Divya Kandpal and Vidhyacharan Bhaskar
}

\begin{abstract}
We consider a system where Generalized Selection Combining (GSC) is applied to a $4 \times \mathrm{L}$ Multiple-Input Multiple-Output (MIMO) system using Half rate-Full diversity Orthogonal Space-Time Block Codes (OSTBCs) at the transmitter. The Symbol Error Rate (SER) of M-ary Quadrature Amplitude Modulation (MQAM) for a slow, flat Rayleigh fading channel is derived and compared to that of the M-ary Phase Shift Keying (MPSK) modulation scheme. Performance comparisons of various antenna configurations are made. The channel statistics are assumed to be identical for the independent diversity branches. The effects of channel estimation errors on the SER performance for GSC are plotted and discussed in this paper.
\end{abstract}

Index Terms-Multiple-input multiple-output (MIMO) systems, channel estimation error, generalized selection combining (GSC), space-time block codes (STBCs), quadrature amplitude modulation (QAM), symbol error rate (SER).

\section{INTRODUCTION}

Diversity schemes provide significant improvement in communication when transmitting through a fading propagation medium. Existing receiver designs implementing the Alamouti transmission scheme used either the Maximal Ratio Combining (MRC) or the Selection Combining (SC) technique [1]. MRC as an optimal combining technique, combines signals from all the receiver branches to maximize Signal-to-Noise Ratio (SNR) at the expense of implementation complexity. The SC scheme, which is the simplest selection method, selects only one receiver branch providing the largest SNR. However, SC provides a poorer system performance than MRC, because the receiver does not fully exploit the available diversity offered by the channel.

The average SNR, outage probability and coding gain were studied in [2] for systems with joint selection at the transmitter and receiver ends. The channel estimate using pilot symbols was derived in [3]. The main problem with the use of diversity schemes is that multiple RF chains associated with multiple antennas are costly to implement. For this reason, there is now a great interest in systems that select a subset of available antennas for reception. Recently, a hybrid version of MRC and SC, called Generalized Selection Combining (GSC) has been proposed and analyzed [4]. GSC selects Ls branches out of $\mathrm{L}$ diversity branches having largest

Manuscript received January 31, 2013; revised April 20, 2013.

The authors are with the Department of Electronics and Communication Engineering at S.R.M. University, Kattankulathur, India (e-mail: divya _ec@rediffmail.com, meetvidhyacharan@yahoo.com) instantaneous SNR and combines them using MRC. Since MRC is sensitive to channel estimation errors, and with GSC, the weak signals which are prone to these errors are excluded in combining GSC with Ls/L (selecting Ls receiver branches out of $L$ available ones), and outperform a MRC scheme with diversity Ls.

Earlier works in GSC were focused on Single-Input Multiple-Output (SIMO) systems without Space-Time Coding (STC) [5]. A virtual branch technique was introduced to derive the mean and variance of the combined output of GSC diversity system. Later, with the aid of STC, diversity gain became possible using multiple transmit antennas. In [6], the distribution theory of transformation of random vectors was discussed. In [7], performance bounds were derived for STBC with GSC at the receiver side.

In [8], the Bit Error Rate (BER) performance of GSC with $L s=2$ and $L s=3$ out of $L$ branches was analyzed, and it was deduced that the expressions became extremely complicated for $L>3$. The effect of channel estimation errors on receiver selection combining schemes for MIMO systems with BPSK was discussed in [9]. The results were extended to include the MPSK case in [10]. The performance of MPSK with GSC in Nakagami fading channel was studied in [11]. The effects of noise and estimator decorrelation on the received BER of multilevel QAM were examined in [12].

In this paper, the effect of channel estimation error on the Symbol Error Rate (SER) performance of a 4 x L MIMO system in a slow, flat Rayleigh fading channel is examined. Coherent detection of MQAM modulation technique is considered, and its performance is compared to that of MPSK modulation scheme. At the transmitter, half rate full diversity OSTBC scheme for complex signal constellations is used. The case of imperfect channel estimation is also considered. GSC requires information regarding SNR of selected branches, and channel estimation information for its operation. Quantitative results for the effects of noisy channel estimation are derived.

This paper consists of five sections. In Section II, the system model is described and OSTBC transmission scheme is introduced. In Section III, a SER expression for GSC reception of MQAM and MPSK in Rayleigh fading channels is derived. In Section IV, numerical results are presented and discussed. Finally, Section V presents the conclusion and future work.

\section{System ModeL}

We consider a MIMO system where an OSTBC scheme is applied with 4 transmits antennas and $L$ receive antennas. 
Fig. 1 shows the system model using an OSTBC transmission scheme and a GSC receiver. Signals $s_{1}, s_{2}, s_{3}$ and $s_{4}$ are sent simultaneously during eight consecutive time slots. The corresponding received signals in these eight intervals on the $i^{\text {th }}$ branch can be expressed as

$$
\left.\begin{array}{c}
r_{1, i}=g_{1, i} s_{1}+g_{2, i} s_{2}+g_{3, i} s_{3}+g_{4, i} s_{4}+n_{1, i}, \\
r_{2, i}=-g_{1, i} s_{2}+g_{2, i} s_{1}-g_{3, i} s_{4}+g_{4, i} s_{3}+n_{2, i}, \\
r_{3, i}=-g_{1, i} s_{3}+g_{2, i} s_{4}+g_{3, i} s_{1}-g_{4, i} s_{2}+n_{3, i}, \\
r_{4, i}=-g_{1, i} s_{4}-g_{2, i} s_{3}+g_{3, i} s_{2}+g_{4, i} s_{1}+n_{4, i}, \\
r_{5, i}=g_{1, i} s_{1}^{*}+g_{2, i} s_{2}^{*}+g_{3, i} s_{3}^{*}+g_{4, i} s_{4}^{*}+n_{5, i}, \\
r_{6, i}=-g_{1, i} s_{2}^{*}+g_{2, i} s_{1}^{*}-g_{3, i} s_{4}^{*}+g_{4, i} s_{3}^{*}+n_{6, i}, \\
r_{7, i}=-g_{1, i} s_{3}^{*}+g_{2, i} s_{4}^{*}+g_{3, i} s_{1}^{*}-g_{4, i} s_{2}^{*}+n_{7, i} \\
r_{8, i}=-g_{1, i} s_{4}^{*}-g_{2, i} s_{3}^{*}+g_{3, i} s_{2}^{*}+g_{4, i} s_{1}^{*}+n_{8, i},
\end{array}\right\}
$$

where $j=1,2, i=1,2, \ldots, L$, and $g_{j, i}$ is the complex gain and represents Additive White Gaussian Noise (AWGN) between the $j^{\text {th }}$ transmit antenna and $i^{\text {th }}$ receive antenna. The variance of real (or imaginary) components of $g_{j, i}$ and $n_{j, i}$ are denoted by $\sigma_{g}^{2}$ and $\sigma_{n}^{2}$, respectively. The average SNR per symbol of the received signal is defined as $\bar{\gamma}=\sigma_{g}^{2} / \sigma_{n}^{2}$.

At the GSC receiver, the received signal from each receive antenna is first processed by a Space-Time (ST) combiner which computes the receiver decision variables as in [1]. Then, the SNR of the output signals is measured and $L_{s}$ out of $L$ signals with largest SNR are selected and combined by a MRC combiner. The combined signal is used for final decision and the signal estimate is based on the phase of the MRC combiner output, $j=1,2$ with equal SNR over receiver branches. The receiver decision variables are computed as

$$
\left.\begin{array}{l}
y_{1, i}=r_{1, i} \hat{g}_{1, i}^{*}+r_{2, i} \hat{g}_{2, i}^{*}+r_{3, i} \hat{g}_{3, i}^{*}+r_{4, i} \hat{g}_{4, i}^{*}+r_{5, i}^{*} \hat{g}_{1, i}+r_{6, i}^{*} \hat{g}_{2, i}+r_{7, i}^{*} \hat{g}_{3, i}+r_{8, i}^{*} \hat{g}_{4, i}, \\
y_{2, i}=r_{1, i} \hat{g}_{2, i}^{*}-r_{2, i} \hat{g}_{1, i}^{*}-r_{3, i} \hat{g}_{4, i}^{*}+r_{4, i} \hat{g}_{3, i}^{*}+r_{5, i}^{*} \hat{g}_{2, i}-r_{6, i}^{*} \hat{g}_{1, i}-r_{7, i}^{*} \hat{g}_{4, i}+r_{8, i}^{*} \hat{g}_{3, i}, \\
y_{3, i}=r_{1, i} \hat{g}_{3, i}^{*}+r_{2, i} \hat{g}_{4, i}^{*}-r_{3, i} \hat{g}_{1, i}^{*}-r_{4, i} \hat{g}_{2, i}^{*}+r_{5, i}^{*} \hat{g}_{3, i}+r_{6, i}^{*} \hat{g}_{4, i}-r_{7, i}^{*} \hat{g}_{1, i}-r_{8, i}^{*} \hat{g}_{2, i}, \\
y_{4, i}=r_{1, i} \hat{g}_{4, i}^{*}-r_{2, i} \hat{g}_{3, i}^{*}+r_{3, i} \hat{g}_{2, i}^{*}-r_{4, i} \hat{g}_{1, i}^{*}+r_{5, i}^{*} \hat{g}_{4, i}-r_{6, i}^{*} \hat{g}_{3, i}+r_{7, i}^{*} \hat{g}_{2, i}-r_{8, i}^{*} \hat{g}_{1, i} \cdot
\end{array}\right\}
$$

where $\hat{g}_{j, i}$ is the estimate of $g_{j, i}$ with variance $\sigma_{\hat{g}}^{2}$, for the real and imaginary part.

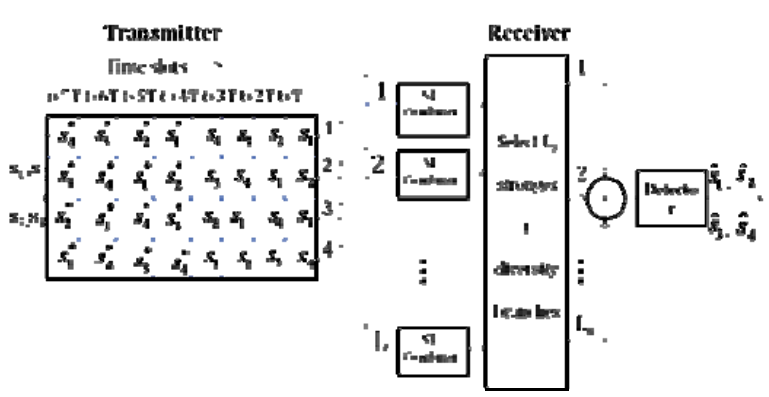

Fig. 1. AMIMO4×L system using half rate full diversity OSTBC and conventional GSC.

The channel estimate is assumed to be a zero-mean complex Gaussian random variable correlated with true channel gain. Extending the results in [2] to include the case when the variances of the channel gain and its estimate are unequal. $g_{j, i}$, is defined as

$$
g_{j, i}=\left(\frac{R_{c}}{\sigma_{\hat{g}}^{2}}+\frac{R_{c s}}{\sigma_{\hat{g}}^{2}}\right) \hat{g}_{j, i}+\left(x_{j, i}+y_{j, i}\right),
$$

where $x_{j, i}$ and $y_{j, i}$ are real Gaussian distributed random variables uncorrelated with $\hat{g}_{j, i}$. The parameters, $R_{c}$ and $R_{c s}$ are given as [2]

$$
\begin{aligned}
& \left.\left.R_{c}=E\left[g_{I} \hat{g}_{I}\right]\right]=E\left[g_{Q} \hat{g}_{Q}\right]\right], \\
& \left.\left.R_{c s}=E\left[g_{I} \hat{g}_{Q}\right]\right]=E\left[g_{Q} \hat{g}_{I}\right]\right] .
\end{aligned}
$$

The true channel gain satisfies, $g_{j, i}=k \hat{g}_{j, i}+d_{j, i}$, where $k=R_{c} / \sigma_{\hat{g}}^{2}$. The channel estimation error, $\rho$, ranges from 0 to 1. The variance of real (or imaginary) component of $g_{j, i}$ is $\sigma_{\mathrm{d}}{ }^{2}$ $=(1-\rho) \sigma_{\mathrm{g}}{ }^{2}$ [3], where $\rho$ is the squared amplitude of the cross-correlation coefficient of the channel fading and its estimate is given by [2]

$$
\rho=\frac{E^{2}[g \hat{g}]}{E\left[|g|^{2}\right] E\left[|\hat{g}|^{2}\right]}=\frac{R_{c}^{2}}{\sigma_{g}^{2} \sigma_{\hat{g}}^{2}}=\frac{\sigma_{\hat{g}}^{2}}{\sigma_{g}^{2}} k^{2} .
$$

For perfect channel estimation, $\rho=1$. When channel estimation deteriorates, we have $\rho \rightarrow 0$.

\section{Symbol ERror Probability of GSC}

The SER for GSC in a multipath fading environment is obtained by averaging the conditional SER over the Probability Density Function (PDF) of the SNR of the MRC combiner output, $P_{e, G S C}$, as

$$
P_{e, G S C}=E_{\gamma_{G S C}}\left[\operatorname{Pr}\left(e \mid \gamma_{G S C}\right)\right]=\int_{0}^{\infty} \operatorname{Pr}(e \mid \gamma) f_{\gamma_{G S C}}(\gamma) d \gamma,
$$

where $\operatorname{Pr}\left(e \mid \gamma_{G S C}\right)$ is the conditional SER.

\section{A. Expression for $\gamma_{\mathrm{GSC}}$}

At the MRC combiner, the selected $\mathrm{L}_{\mathrm{s}} \mathrm{ST}$ combiner outputs are combined. Defining the effective SNR as

$$
\bar{\gamma}_{c=} \frac{\rho \bar{\gamma}}{(1-\rho) \bar{\gamma}+1}
$$

The sum of the SNR of selected branches is given as

$$
\gamma_{G S C}=\frac{\bar{\gamma}_{C}}{2} \sum_{i=1}^{L_{S}} a_{i},
$$

where $a_{[L]}$ is an ensemble of $a_{i}$ with $a_{1}>a_{2} \ldots>a_{L}$ which is the column vector represented as $a_{[L]}=\left[\begin{array}{llll}a_{1} & a_{2} & \cdots & a_{L}\end{array}\right]^{T}$.

\section{B. Derivation of SER}

The independent random variable $a_{i}$ has a chi-square distribution with four degrees of freedom and its PDF is given by

$$
f\left(a_{i}\right)=a_{i} \exp \left(-a_{i}\right) \forall a_{i}>0
$$


The joint PDF of the ordered set in GSC can be expressed as [5]

$$
f_{a_{[L]}}=L ! f\left(a_{1}\right) f\left(a_{2}\right) \ldots f\left(a_{L}\right), a_{1}>a_{2} \ldots .>a_{L} .
$$

The ordered $a_{[L]}$ are transformed into a new set of independent variables, $\mathrm{v}_{[\mathrm{L}]}$ using virtual branch relation $\mathrm{a}_{[\mathrm{L}]}=\mathrm{T}_{\mathrm{VB}} \mathrm{V}_{[\mathrm{L}]}$, where $T_{V B}$ is given as [5]

$$
T_{V_{B}}=\left[\begin{array}{cccc}
1 & \frac{1}{2} & \cdots & \frac{1}{L} \\
0 & \frac{1}{2} & \cdots & \frac{1}{L} \\
0 & 0 & \ddots & \vdots \\
0 & 0 & 0 & \frac{1}{L}
\end{array}\right] .
$$

Substituting (9) into (10), we have

$$
f_{a_{[L]}}=L ! \prod_{i=1}^{L} a_{i} \exp \left(-\sum_{i=1}^{L} a_{i}\right), a_{1}>a_{2} \cdots>a_{L} .
$$

Using the distribution theory for transformation of random vectors [6]

$$
f_{v_{[L]}}\left(v_{1}, v_{2}, \ldots, v_{L}\right)=J f_{a_{[L]}}\left(a_{1}, a_{2}, \ldots, a_{L}\right),
$$

where $J$ is the Jacobian of the virtual branch transformation, from (11), we have

$$
J=\left|T_{V B}\right|=\prod_{n=1}^{L} \frac{1}{n}=\frac{1}{L !} .
$$

Substituting (14) into (13) and using the distribution theory of transformation of random vectors [6], the joint PDF of $\mathrm{V}_{[\mathrm{L}]}$ is given by

$$
f_{v_{[L]}}\left(v_{1}, v_{1}, \cdots, v_{L}\right)=\prod_{i=1}^{L} a_{i} \exp \left(-\sum_{i=1}^{L} v_{i}\right) .
$$

For coherent detection of MQAM, the conditional SER expression is given by [5]

$$
\begin{array}{r}
\operatorname{Pr}\left(e_{M Q A M} \mid \gamma_{G S C}\right)=\frac{q}{\pi} \int_{0}^{\pi / 2} \exp \left(-\frac{c_{M Q A M}}{\sin ^{2} \theta} \gamma_{G S C}\right) d \phi \\
-\frac{q^{2}}{4 \pi} \int_{0}^{\pi / 4} \exp \left(-\frac{c_{M Q A M}}{\sin ^{2} \theta} \gamma_{G S C}\right) d \varphi,
\end{array}
$$

where $_{q=4}\left(1-\left(\frac{1}{\sqrt{M}}\right)\right)$ and $c_{M Q A M}=\frac{3}{2(M-1)}$.

Substituting (15) and (16) into (6), and solving for $L=2, L_{s}$ $=1$ and QAM modulation, we have

$$
\begin{aligned}
P_{e, G S C}= & \frac{7}{24}\left[\frac{2}{\pi} \int_{0}^{\pi / 2} \frac{1}{\left(\frac{0.25 \bar{\gamma}}{\sin ^{2} \phi}+1\right)\left(\frac{0.25 \bar{\gamma}}{2 \sin ^{2} \phi}+1\right)} d \phi-\right. \\
& \left.\frac{1}{\pi} \int_{0}^{\pi / 4} \frac{1}{\left(\frac{0.25 \bar{\gamma}}{\sin ^{2} \varphi}+1\right)\left(\frac{0.25 \bar{\gamma}}{2 \sin ^{2} \varphi}+1\right)} d \varphi\right] .
\end{aligned}
$$

Similarly, SER can be derived for higher order modulation schemes $(M=8,16,32)$ and multiple receive antennas $(L=3$, $4,5)$. For coherent detection of MPSK, conditional SER is written as [5]

$$
\operatorname{Pr}\left(e \mid \gamma_{G S C}\right)=\frac{1}{\pi} \int_{0}^{\theta} \exp \left(-K(\phi) \gamma_{G S C}\right) d \phi
$$

where $K(\phi)=\frac{\sin ^{2}(\pi / M)}{\sin ^{2} \phi}$ and $\theta=\pi(M-1) / M$

Substituting (15) and (18) into (6), the SER expression for $\mathrm{L}=2, \mathrm{~L}_{\mathrm{s}}=1$ case with QPSK modulation is given as

$$
\begin{aligned}
P_{e, G S C}=\frac{1}{\pi} \int_{0}^{\infty} \int_{0}^{\infty} \int_{0}^{3 \pi / 4} \exp \left[-\frac{0.5 \bar{\gamma}}{2 \sin ^{2} \phi}\left(v_{1}+\frac{v_{2}}{2}\right)\right] \\
\left(v_{1}+\frac{v_{2}}{2}\right)\left(\frac{v_{2}}{2}\right) \exp \left(-v_{1}-v_{2}\right) d \varphi d v_{2} d v_{1} .
\end{aligned}
$$

The integral in (19) can be further simplified to

$$
P_{e, G S C}=\frac{7}{24 \pi} \int_{0}^{3 \pi / 4} \frac{1}{\left(\frac{0.25 \bar{\gamma}}{\sin ^{2} \phi}+1\right)\left(\frac{0.25 \bar{\gamma}}{2 \sin ^{2} \phi}+1\right)} d \phi .
$$

Similarly SER expression can be derived for multiple receive antennas $(L=3,4,5)$.

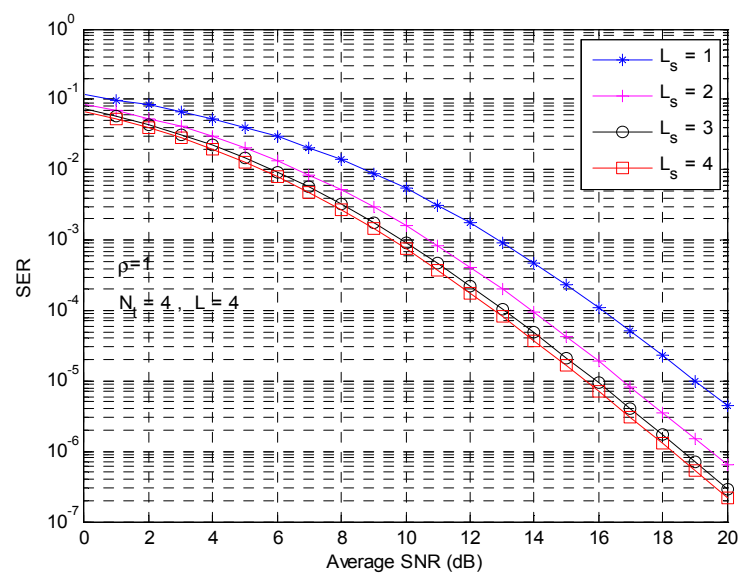

Fig. 2. SER of QPSK vs. SNR per symbol for $4 \times 4$ antenna system with perfect channel estimation.

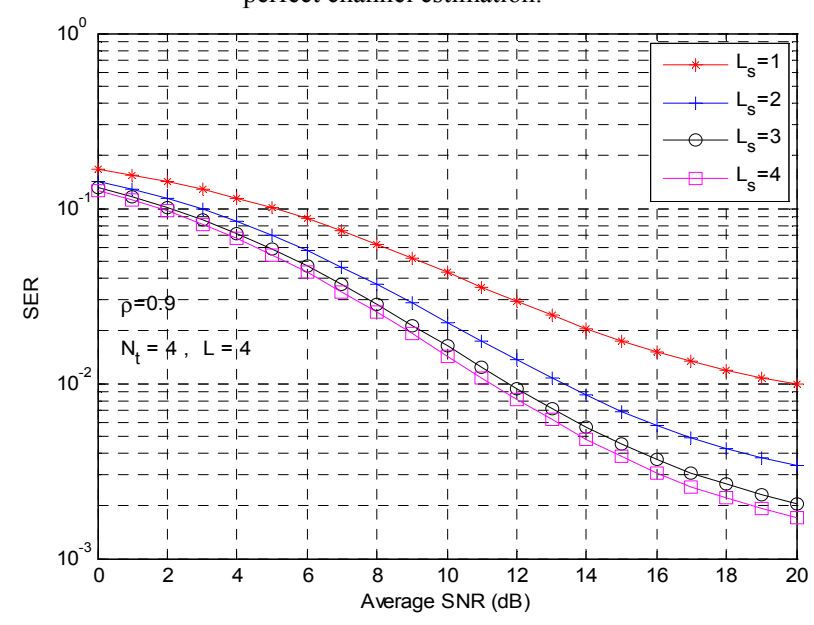

Fig. 3. SER of QPSK vs. SNR per symbol for $4 \times 4$ antenna system with imperfect channel estimation $(\rho=0.9)$.

\section{Simulation Results}

As expected, the simulation results show that SER increases with increasing fading estimation error (decreasing value of $\rho$ ). Fig. 2 shows that system performance improves as more antennas are selected at the receiver. As $L_{s}$ increases, 
the system performance improves because output SNR of GSC is the sum of SNR of selected branches.

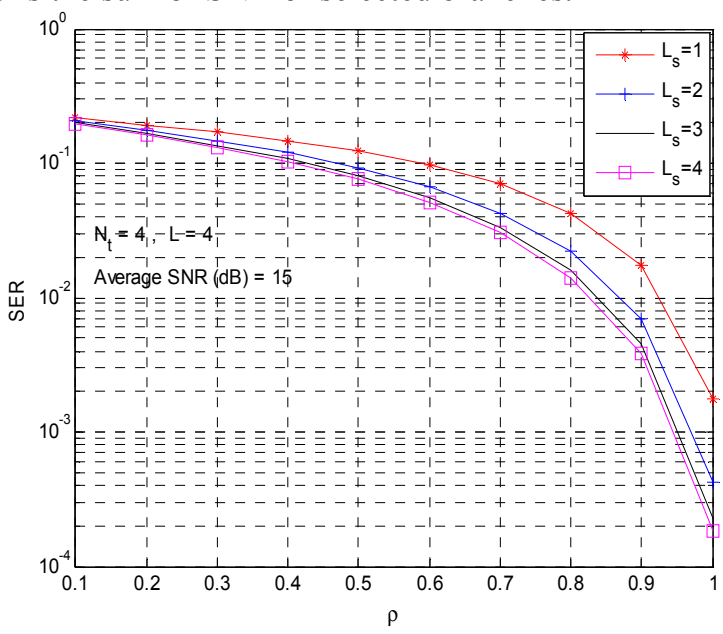

Fig. 4. SER of QPSK vs. $\rho$ for $4 \times 4$ antenna system with average SNR per symbol $=15$.

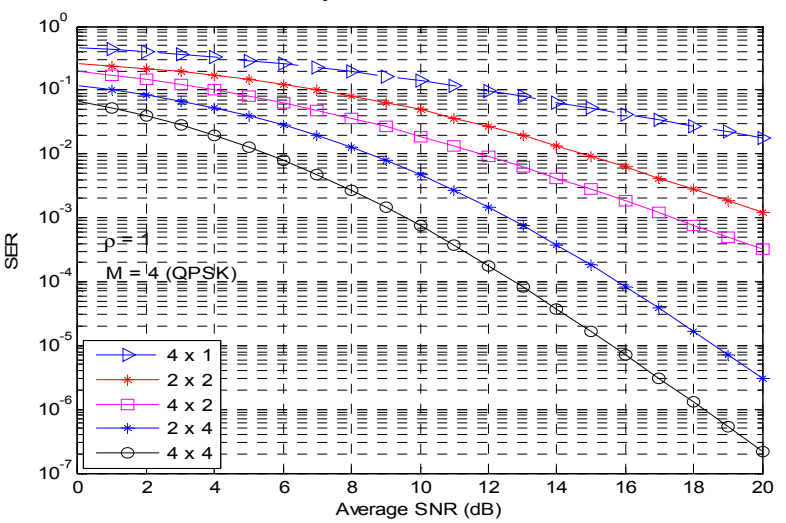

Fig. 5. SER vs. Average SNR of QPSK fordifferent antenna configurations with perfect channel estimation $(\rho=1)$

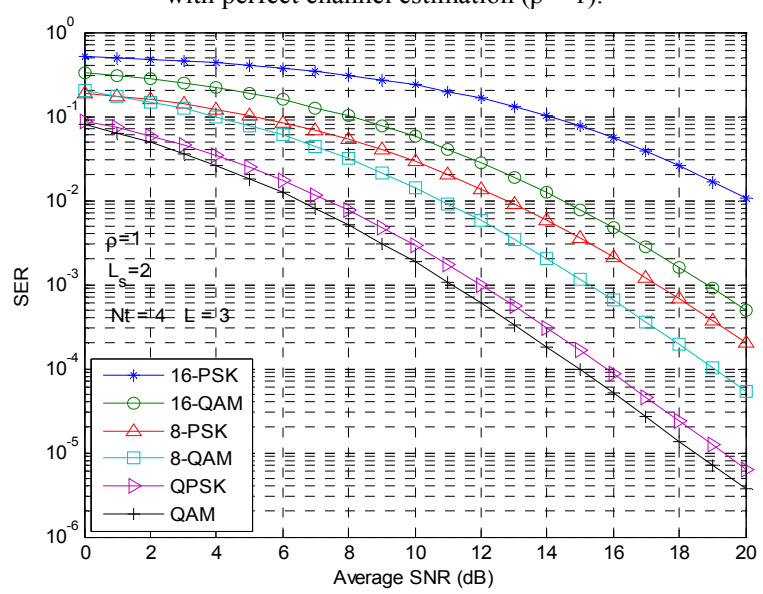

Fig. 6. Performance comparison of MQAM and MPSK for $4 \mathrm{~T} \times$ and $3 \mathrm{Rx}$ system with perfect channel estimation $(\rho=1)$.

Fig. 3 shows similar performance comparison for imperfect channel estimation case. Fig. 4 shows that the performance of $4 \times 4$ half-rate full diversity OSTBC degrades with imperfection in the channel, but improves with increase in the number of selected signals. Performance comparison of different antenna configurations is shown in Fig. 5. It shows that the $4 \times 4$ MIMO system performs better than the 2 $\times 2$ antenna transmission system. The effect of increasing the number of receive antennas is greater than the effect of increasing the number of transmit antennas. Fig. 6 shows that the performance of QAM modulation scheme is better than that of the QPSK modulation scheme, and the performance degrades with increase in modulation order.

\section{CONCLUSIONS}

The effect of channel estimation errors on SER performance of half-rate full diversity OSTBC for a $4 \times L$ antenna system with GSC technique was illustrated in this paper. It can be observed that performance degrades with imperfection in channel estimation (decrease in value of parameter $\rho$ ). Analytical results were derived for $4 \times L$ MIMO systems using M-QAM modulation schemes in Rayleigh fading channels. The system performance was shown to improve with increase in diversity order. Further, increase in the number of transmit antennas for a full rate full diversity OSTBC is more interesting and challenging, and will be the focal point of future research work.

\section{REFERENCES}

[1] M. Alamouti, "A simple transmit diversity technique for wireless Communications," IEEE Journal on Selected Areas in Communications, vol. 16, no. 8, pp. 1451-1458, Oct. 1998.

[2] M. J. Gans, "The effect of Gaussian error in maximal ratio combiners," IEEE Transactions on Communications, vol. 19, no. 4, pp. 492-500, Aug. 1971.

[3] D. A. Gore and A. J. Paulraj, "MIMO antenna subset selection with space-time coding," IEEE Transactions on Signal Processing, vol. 50, no. 10 , pp. 2580-2588, Oct. 2002.

[4] M. S. Alouini and M. K. Simon, "An MGF-based performance analysis of generalized selection combining over Rayleigh fading channels," IEEE Transactions on Communications, vol. 48, no. 3, pp. 401-415, March. 2000.

[5] M. Z. Win and J. H. Winters, "Virtual branch analysis of symbol error probability for hybrid selection/maximal-ratio combining in Rayleigh fading channels," IEEE Transactions on Communications, vol. 49, no. 11, pp. 1926-1934, Nov. 2001.

[6] P. J. Bickel, and K. Doksum, Mathematical Statistics: Basic Ideas and Selected Topics, 1st ed. Oakland, CA: Holden-Day, 1977.

[7] X. Zeng and A. Ghrayeb, "Performance bounds for space-time block codes with receive antenna selection," IEEE Transactions on Information Theory, vol. 50, no. 9, pp. 2130-2137, Sep. 2004.

[8] T. Eng, N. Tong, and L. B. Milstein, "Comparison of diversity combining techniques for Rayleigh-fading channels," IEEE Transactions on Communications, vol. 44, no. 9, pp. 1117-1129, Sep. 1996.

[9] W. Li, and C. Beaulieu, "Effects of channel estimation errors on receiver selection combining diversity for Alamouti MIMO systems with BPSK," IEEE Transactions on Communications, vol. 54, no.1, pp. 169-178, Nov. 2006.

[10] W. Li and C. Beaulieu, "Generalized receiver Selection Combining Schemes for Alamouti MIMO Systems with MPSK," IEEE Transactions on Communications, vol. 57, no. 6, pp. 1599-1602, June 2009.

[11] Y. Ma, R. Schober, and S. Pasupathy, "Performance of MPSK with GSC and EGC with Gaussian weighting errors," IEEE Transactions on Vehicular Technology, vol. 54, no. 1, pp. 149-162, Jan. 2005.

[12] L. Cao and N. C. Beaulieu, "Exact error-rate analysis of diversity 16-QAM with channel estimation error," IEEE Transactions on Communications, vol. 52, no. 6, pp. 1019-1029, June 2004.

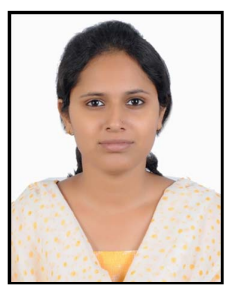

Divya Kandpal received the B. Tech. degree in Electronics and Communication Enginnering from Kumaon Engineering College, Dwarahat, India in 2010 and the M. Tech. degree in Communication Systems from SRM University, Chennai, India in 2012. Her research interests include wireless communications and Multiple-Input Multiple-Output (MIMO) antenna systems. She is currently with Uttarakhand Power Corporation Limited, India. 


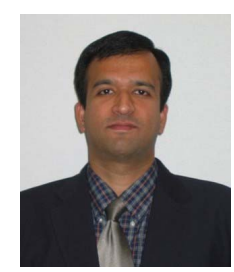

Vidhyacharan Bhaskar received the B.Sc. degree in Mathematics from D.G. Vaishnav College, Chennai, India in 1992, M.E. degree in Electrical \& Communication Engineering from the Indian Institute of Science, Bangalore in 1997, and the M.S.E. and Ph.D. degrees in Electrical Engineering from the University of Alabama in Huntsville, USA in 2000 and 2002 respectively. During 2002-2003, he was a Post-Doctoral fellow with the Wireless Communications research group at the University of Toronto, Canada. From Sep. 2003 to Dec. 2006 , he was an Associate Professor in the Département of Information Systems and Telecommunications at the University of Technology of Troyes, France. Since January 2007, he is a Professor of the Department of Electronics and Communication Engineering at S.R.M. University, Kattankulathur, India. His research interests include Wireless Communications, Signal processing, Error control coding and Queuing theory.
He has published 73 refereed Journal papers and 43 Conference papers in various International Conferences. Besides this, he also has around 10 Journals currently under review. He has also published two text books, one, on "Adaptive Rate Coding for A-CDMA Systems", in January 2011 published by LAP Lambert Publishing agency, Germany, and the other on "MATLAB FOR ALL" in USA in 1998. He has Supervised 3 PhD research scholars, and 33 Masters students. In Sep. 2012, he won the IETE Prof. SVC Aiya Memorial award for Outstanding Contributions in the area of Electronics and Telecommunications in 2012 from Institute of Electronics and Telecommunication Engineers (IETE). He is also a Fellow of the IETE In Jan. 2012, he won the award for the "Best Academic Researcher" from the Association of Scientists, Developers, and Faculties associated with the Techno Form Research and Development Group, Pondicherry. 